\title{
DÉTECTION DE L'ÉROSION DANS UN BASSIN VERSANT AGRICOLE PAR COMPARAISON D'IMAGES MULTIDATES ACQUISES PAR DRONE
}

\author{
Jonathan Lisein ${ }^{1,2}$, Nathalie Pineux ${ }^{3}$, Marc Pierrot-Deseilligny ${ }^{2,4}$, Aurore Degré ${ }^{3}$, Philippe Lejeune ${ }^{1}$ \\ 1: Université de Liège - Gembloux Agro-Bio Tech. Unité de Gestion des Ressources forestières et des Milieux naturels. 2, Passage des \\ Déportés, 5030 Gembloux, Belgique \\ 2: Université Paris-Est (UPE), 6 et 8 avenue Blaise Pascal - Cité Descartes - Champs-sur-Marne - 77455 Marne la Vallée, France \\ 3: Université de Liège - Gembloux Agro-Bio Tech. Unité d'Hydrologie et hydraulique agricole. 2, Passage des Déportés, 5030 Gembloux, \\ Belgique \\ 4: Institut national de l'information géographique et forestière (IGN)/SR/LOEMI - 4, avenue Pasteur - 94165 Saint-Mandé cedex, France
}

\begin{abstract}
Résumé
L'émergence des drones comme outils de cartographie rapide, de par leur capacité à répondre à des besoins très spécifiques, offre de nombreuses opportunités aux scientifiques. Par ailleurs, les récentes évolutions des techniques de photogrammétrie et de vision par ordinateur permettent, à partir de prises de vues aériennes stéréoscopiques, de fournir aux géomorphologues et aux hydrologues des données topographiques à haute résolution (Tarolli, 2014). En effet, les algorithmes d'orientation externe (structure from motion en anglais, Snavely et al. (2008)) permettent la détermination automatique de la position et de l'orientation des prises de vue d'une collection d'images se recouvrant. La corrélation dense automatique permet ensuite, depuis un bloc d'images orientées, de modéliser finement le relief. L'utilisation en géomorphologie de drones pour la modélisation du relief en est encore à ses premiers souffles, mais montre un potentiel très intéressant. La précision des mesures photogrammétriques rivalise en effet avec les relevés LiDAR, pour un coût d'acquisition significativement moins élevé. Cette recherche se focalise sur deux objectifs. Le premier est de déterminer si la précision des mesures photogrammétriques issues d'images acquises avec un mini-drone permet la détection de changement de relief très fin via la comparaison d'acquisitions multi-dates. Le deuxième objectif, plus spécifique, est de déterminer la manière la plus optimale de paramétrer la compensation par faisceaux avec points d'appui au sein de la suite photogrammétrique open-source MICMAC.
\end{abstract}

Mots clés : Drone, Photogrammétrie, Bassin versant, Érosion, Compensation par faisceaux, logiciel MICMAC

\section{Introduction}

Le regain d'intérêt pour les techniques de photogrammétrie et de vision par ordinateur auquel on assiste depuis quelques années s'explique par l'amélioration en matière de puissance de calcul des ordinateurs et par l'omniprésence des appareils photographiques. Grâce à ces algorithmes de photogrammétrie, une caméra amateur embarquée sur un avion sans pilote se transforme en un outil de mesure permettant la modélisation très fine de la topographie de la scène survolée. De nombreuses recherches relatent la récente effervescence autour de l'apport de la photogrammétrie terrestre (Westoby et al. 2012, rapprochée (Fonstad et al., 2013) ou par drone dans la compréhension des phénomènes hydrologiques et topographique (Colomina et Molina 2014; Tarolli 2014). La comparaison avec des données LiDAR est très souvent au centre du débat, cette dernière étant considérée comme $L A$ référence dans bien des domaines en matière de données tridimensionnelles.

Dans les paysages agricoles, la microtopographie du sol est en changement permanent, notamment dû à l'érosion et aux dépôts. La solution pour lutter contre ces augmentations de flux (provoquant la variation de qualité des cours d'eau et l'augmentation des inondations) et cette perte en terre est de comprendre et localiser ces phénomènes, d'où l'utilité de multiplier les outils pour mesurer ces phénomènes. Mesurer l'érosion et le dépôt est en effet une demande récurrente de la part des politiques et des programmes de conservation et de régulation. Cette mesure est en effet nécessaire pour déterminer l'impact environnemental de l'érosion et des pratiques de conservation, pour le développement de technologies de prévision d'érosion et pour le développement et l'évaluation des mesures de contrôle de l'érosion (Stroosnijder. 2005). Comprendre ces processus physiques permet d'améliorer nos connaissances de l'évolution du paysage à l'échelle temporelle et spatiale Slattery et al. 2002, Saavedra. 2005). L'observation traditionnelle de ces changements de topographie se fait par la pose de jalons de référence, le théodolite, le LiDAR terrestre (Barneveld et al. 2013: Brubaker et al. 2013) ou la photogrammétrie terrestre (Warner 1995). Mais ces observations ne peuvent s'effectuer que sur de petites zones alors que l'échelle la plus appropriée pour comprendre ces phénomènes semble être le bassin versant. De plus, il s'agit de méthodes de contact, perturbant dans une certaine mesure la surface du sol, alors qu'elles ont pour fonction une étude qui requiert des enregistrements répétés dans le 
temps.

La photogrammétrie peut donc être une alternative bon marché et moins consommatrice en temps, permettant une couverture spatiale continue sans une densité d'échantillonnage limitée, et ce de manière non destructive (Hugenholtz et al., 2012, Colomina et Molina, 2014). L'application des drones engendre un intérêt croissant dans le domaine des géosciences. D'abord adopté depuis quelques années dans des études de morphologie côtière ou fluviale (Lejot et al., 2007; Mancini et al., 2013, Javernick et al. 2014), il est finalement utilisé pour des études concernant les glissements de terrain (Niethammer et al. 2012; Stumpf et al. 2013; Lucieer et al. 2014) , des paysages de badlands ou des zones d'érosion en ravine (d'Oleire Oltmanns et al., 2012, Peter et al., 2014). Des invesitigations restent cependant nécessaires afin de mieux quantifier les performances de la photogrammétrie par drone en terme de précision de mesures $(\mathrm{Hu}-$ genholtz et al. 2012). Cette précision dépend de nombreux facteurs, tels que la configuration du bloc d'images (recouvrement, agencement), la calibration de la caméra et le type de surface faisant l'objet de mesures.

Le défi de cette recherche est de spatialiser les phénomènes dans des paysages où les changements de relief sont peu marqués et de dégager des répartitions spatiales d'érosion et de dépôt. La zone d'étude est un bassin versant situé à Haute-Heuval, en Belgique. Ce bassin versant est recouvert à $98 \%$ par des terres agricoles et présente un faible pourcentage de territoire boisé. Un suivi des précipitations est effectué dans le cadre du projet GISER (Gestion intégrée sol- érosion - ruissellement). Une série temporelle d'images acquises par drone est utilisée pour la génération de MNTs à très haute résolution. L'évolution du relief est ensuite mise en évidence par la comparaison des MNTs des différentes dates. Les traitements photogrammétriques sont effectués avec la boîte à outils MICMAC et une attention particulière est portée à l'utilisation des points d'appui dans la compensation par faisceaux afin d'éviter les distorsions non-linéaires dans le modèle d'aérotriangulation (Ouédraogo et al. 2014, $\mathrm{Wu}, 2014$.

\section{Matériel et méthode}

\subsection{Le vecteur et le capteur}

Le petit avion sans pilote utilisé est un drone à voilure fixe, le Gatewing X100 (http ://uas.trimble.com/X100). Son endurance de vol est de maximum 45 minutes pour une vitesse de croisière de $80 \mathrm{~km} / \mathrm{h}$. La navigation de ce drone de $2 \mathrm{~kg}$ s'effectue exclusivement en vol automatique, ce qui fait la simplicité de ce système. Un lanceur (voir figure 1 est utilisé pour le décollage et l'atterrissage s'effectue sur le ventre. La préparation du plan de vol nécessite la définition d'une zone rectangulaire à scanner, d'une hauteur de vol au dessus du niveau du sol et d'une valeur unique de recouvrement d'image faisant office de recouvrement latéral et longitudinal. La localisation et la direction de l'atterrissage doivent également être définis dans le plan de vol. Les vols s'effectuent hors vue. Ce drone

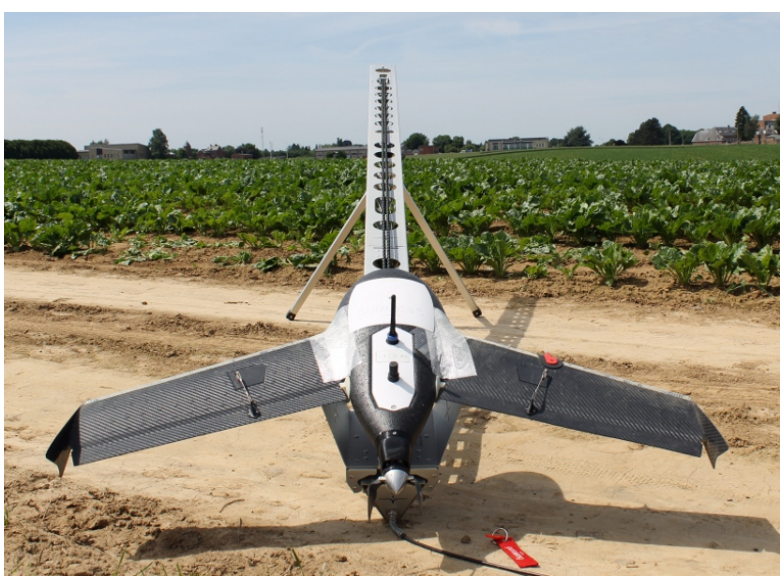

FIGURE 1: Le mini drone Gatewing X100 sur sa rampe de lancement.

a fait ses preuves; notre équipe de pilotes à distance comptabilisent quelques 230 vols effectués avec succès entre 2011 et 2014. Le capteur embarqué est un appareil digital compact de modèle Ricoh GR3 (capteur photographique CCD de 10 megapixels et distance focale équivalente en $35 \mathrm{~mm}$ de $28 \mathrm{~mm}$ ). Cet appareil a été modifié pour les acquisitions dans le proche infrarouge (filtre longue bande jaune pour bloquer le bleu) dans le but initial d'être utilisé à des fins de cartographie de la végétation. L'appareil est réglé en mode manuel et la sensibilité ISO ainsi que la vitesse d'obturation sont définies juste avant le décollage. L'appareil est pré-calibré au moyen d'un polygone d'étalonnage, mis au point et présenté par Tournadre et al. (2014), et les distorsions sont modélisées au moyen d'un modèle de Fraser.

\subsection{Les mesures terrain des points d'appui}

Pour chaque acquisition, une dizaine de cibles sont placées sur le bassin versant et leur position mesurée au moyen d'un GPS (Global Positionning System) Leica GPS1200 en mode RTK (Real Time Kinematic) présentant une précision nominale de $1 \mathrm{~cm}$ en planimétrie et de $1.5 \mathrm{~cm}$ en altimétrie. Le système de projection géographique utilisé pour cette recherche est le Belge Lambert 72 (EPSG :31370). Outre pour le géoréférencement du modèle d'aérotriangulation, les points d'appui sont également utilisés dans le calcul de l'orientation du bloc d'images. Leur utilisation dans la compensation par faisceaux permet de contraindre le modèle et d'estimer correctement l'orientation interne (calibration), sans quoi des distorsions non-linéaires entachent le modèle d'aérotriangulation (Ouédraogo et al. 2014). La disposition et le nombre de points d'appui diffèrent d'une acquisition à l'autre, comme l'illustrent les figures 35

\subsection{Les acquisitions}

Trois acquisitions sont effectuées avec un intervalle de temps d'une année entre les acquisitions successives, soit respectivement en hiver $2011(t 1)$, en hiver 2012 ( $t 2)$ et en hiver $2013(t 3)$. Les acquisitions se sont déroulées en hiver lorsque la couverture végétale est presque nulle, 


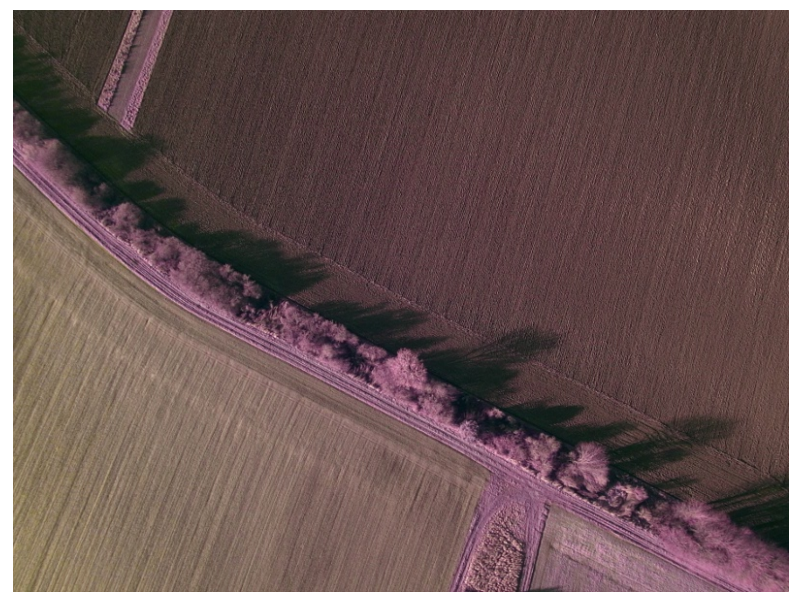

FIGURE 2: Une des images de $6.75 \mathrm{~cm}$ de résolution acquise à une hauteur de vol de 200 mètres.

afin de distinguer la plus grande partie de la surface de sol sur les images. Néanmoins, des intercultures (moutarde) sont présentes sur certaines parcelles agricoles. Les haies et bandes herbeuses empêchent également la visualisation du sol sur l'entièreté du bassin versant. La taille du bassin versant de plus de 200 ha ainsi que la présence à proximité d'une antenne de télécommunication d'une hauteur de 160 mètres ont guidé notre choix de voler à une altitude de 200 mètres au-dessus du point de décollage. La résolution moyenne des images brutes en résultant est de $6.75 \mathrm{~cm} /$ pixel (figure 2). La surface du bassin versant est telle qu'un unique vol ne permet pas de couvrir son entièreté avec un recouvrement d'images suffisant. Deux vols successifs sont donc effectués pour chacune des acquisitions de la série temporelle. Les figures 35 illustrent la configuration des blocs d'images pour chacune des trois acquisitions. Les positions de prises de vue en bleu sont celles du premier vol et en rouge celles du deuxième vol. La direction des lignes de vol est choisie, si possible, de manière à être perpendiculaire au vent dominant, afin d'économiser l'énergie nécessaire au drone pour voler contre le vent. Lors de la seconde acquisition, le vent soufflait depuis le nord, les lignes de vol sont donc orientées est-ouest tandis qu'elles sont orientées nord-sud pour les acquisitions $t 1$ et $t 2$. Outre ces différences, on note que la disposition des points d'appui est la moins optimale pour l'acquisition $t 2$ : ceux-ci sont effectivement répartis trop au centre du bassin versant.

Pour chacun des vols, les autorisations nécessaires ont été délivrées par la Direction Générale des Transports Aériens ainsi que par la commune survolée et par le propriétaire du terrain de décollage et d'atterrissage.

\subsection{Les traitements photogrammétriques}

Pour chaque année, le bloc d'images est utilisé pour délivrer un modèle numérique de terrain à haute résolution. Les traitements d'images acquises par drone sont effectués avec la chaine photogrammétrique open source
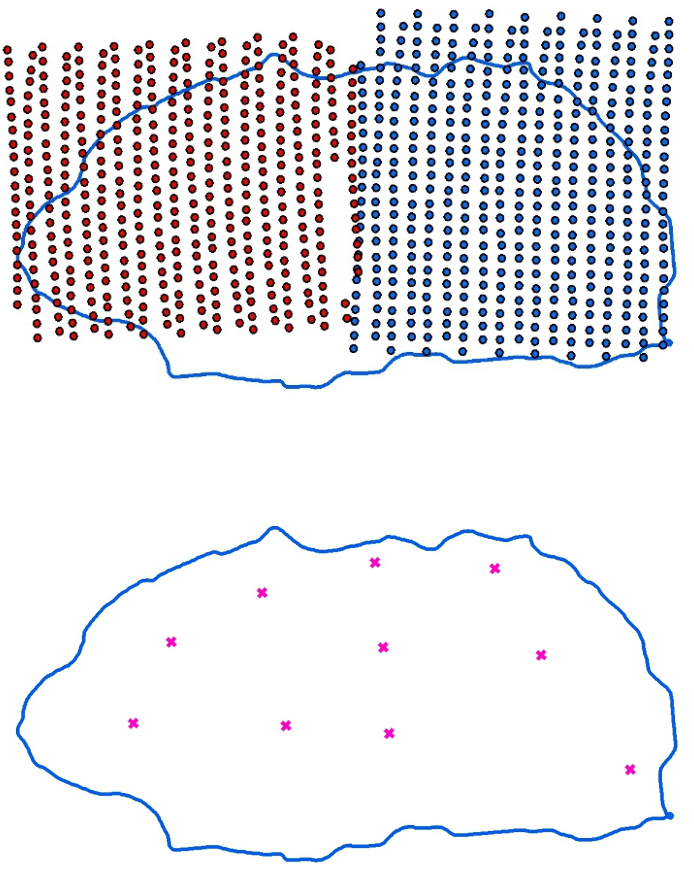

FIGURE 3: Disposition des images (haut) et des points d'appui (bas) pour la première acquisition $(t 1)$. Le bassin versant est couvert en deux vols successifs (différenciés par la couleur des positions des caméras. Le recouvrement est de $80 \%$, le nombre d'images de 868 et le nombre de points d'appui de 10.

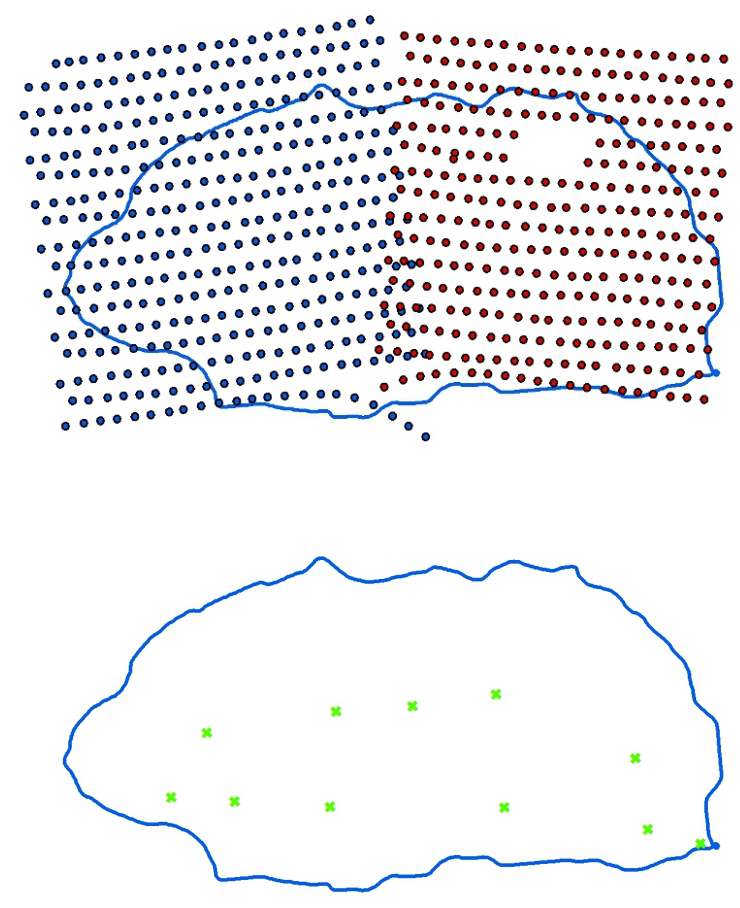

FIGURE 4: Disposition des images (haut) et des points d'appui (bas) pour la deuxième acquisition ( $t 2$ ). Le bassin versant est couvert en deux vols successifs (différenciés par la couleur des positions des caméras. Le recouvrement est de $75 \%$, le nombre d'images de 685 et le nombre de points d'appui de 11 . 

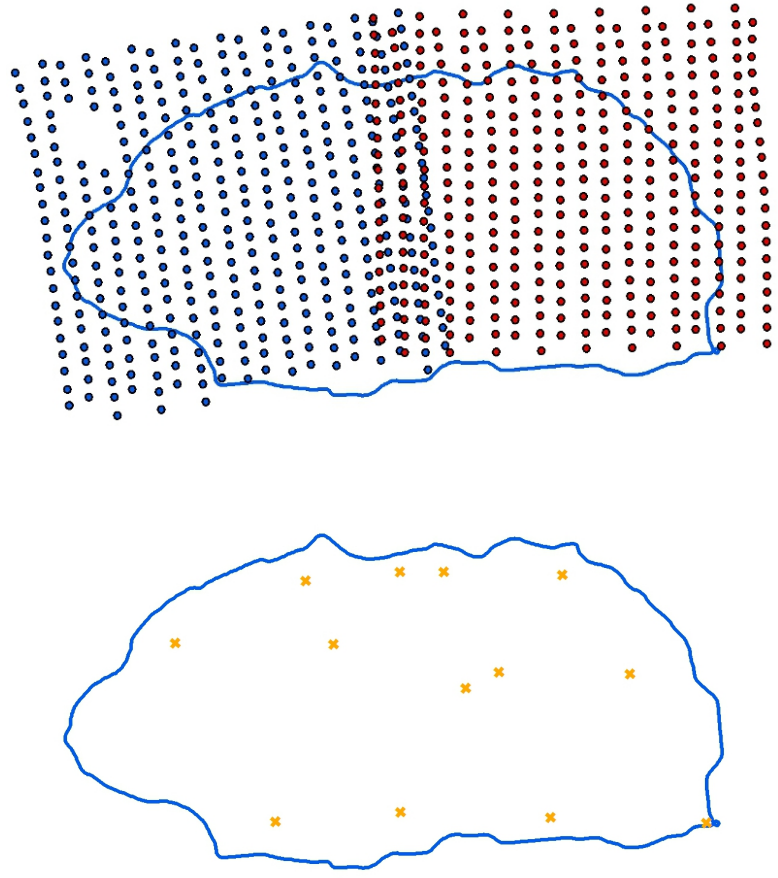

FIGURE 5: Disposition des images (haut) et des points d'appui (bas) pour la deuxième acquisition ( $t 3$ ). Le bassin versant est couvert en deux vols successifs (différenciés par la couleur des positions des caméras. Le recouvrement est de $75 \%$, le nombre d'images de 736 et le nombre de points d'appui de 13 .

Interface graphique

\section{Ligne de commande}

Fichiers $<X M L>$

\section{Code $\mathrm{C}++$}

FIgURE 6: La suite MICMAC est organisée en différentes couches d'une complexité croissante. Les outils complexes se présentent sous la forme de fichier XML contenant leurs paramétrages et les outils simplifiés sont des outils en ligne de commande avec un nombre réduits d'arguments. de l'IGN"1 MICMAC (Pierrot-Deseilligny et Paparoditis 2006. Cette boîte à outils photogrammétrique en ligne de commande est organisée en plusieurs couches, de la couche bas niveau (le code $C++$ ) aux outils simplifiés en ligne de commandes (voir figure 6). L'interface graphique existe déjà mais fait actuellement l'objet de nombreux développements. Les étapes de détermination des points homologues et de corrélation multi-images en multi-résolution sont effectuées avec les outils simplifiés de la suite MICMAC, à savoir respectivement Tapioca et Malt. L'outil complexe d'orientation et de calibration Apero (Pierrot-Deseilligny et Clery 2011; Remondino et al., 2012 est quant à lui utilisé pour paramétrer la compensation par faisceaux avec point d'appui. L'orientation est premièrement calculée dans un système relatif, uniquement sur base des points homologues, et est ensuite géoréférencée et réajustée en ajoutant les points d'appui à la compensation par faisceaux.

Des résultats antérieurs (Lisein et al. 2013) nous ont amenés à utiliser la compensation par faisceaux en autocalibration. Les trois blocs d'images de la série temporelle $t 1, t 2$ et $t 3$ sont orientés de manière indépendante. Au vu du nombre relativement faible de points de contrôle disponibles, une approche de validation croisée est mise en place : un à un, les points de contrôle sont utilisés individuellement comme points de vérification, tandis que les autres sont utilisés comme points d'appui. La racine carrée de la somme des carrés des résidus sur les points de vérification (écart-type) est utilisée comme indicateur de qualité de l'aérotriangulation, mais l'orientation utilisée in fine pour la corrélation dense est celle calculée en utilisant l'ensemble des points de contrôle comme points d'appui.

L'algorithme de compensation par faisceaux implémenté dans Apero permet un contrôle fin des différentes étapes de la compensation (qui est un processus itératif, le système d'équation étant un système non-linaire surabondant). En particulier, la pondération des différents types d'observation utilisée dans le calcul de l'orientation et la gestion des observations aberrantes à fait l'objet de divers tests. Les mécanismes de pondération des mesures de points homologues, des mesures terrain des points d'appui et des mesures images des points d'appui (marquage des cibles sur les images) sont décrites dans la documentation de MICMAC (Pierrot-Deseilligny 2014) à laquelle nous renvoyons le lecteur désirant une description plus détaillée de ces mécanismes. Le paramétrage par défaut de la compensation par faisceaux de la suite MICMAC est particulièrement adapté à la photogrammétrie traditionnelle, à savoir celle utilisant des images acquises au moyen d'un capteur métrique et dont la disposition dans le bloc d'images est régulière avec une prise de vue quasiment verticale Colomina et Molina, 2014). Les blocs d'images acquis par drone diffèrent de ceux de la photogrammétrie traditionnelle, suite à la

1. L'institut national de l'information géographique et forestière 
mauvaise stabilité géométrique de la caméra, la faible netteté des images et les fortes variations d'orientation de l'appareil photographique dues à l'instabilité du drone provoquée par sa prise au vent. II s'est avéré nécessaire de modifier la pondération des mesures des points d'appui (les mesures terrain d'une part et les mesures images d'autre part), car les résultats avec la pondération par défaut montrent des résidus aberrants tant pour les mesures images que pour les mesures terrain des points d'appui. En effet, pour nos blocs d'images, trop d'importance est donnée par défaut aux mesures terrain des points d'appui. Les résidus sur ces observations sont donc très faibles; de l'ordre du $\mathrm{mm}$ alors que la précision de mesure est de 1 à $2 \mathrm{~cm}$. Les mesures images des points d'appui montrent, au contraire, un résidu dont la valeur dépasse 5 pixels. Le marquage de nos points de contrôle sur les images s'effectue avec une précision de l'ordre de 1 à 2 pixels. Ces résidus trop élevés nous ont donc amenés à la conclusion qu'il était nécessaire d'augmenter la pondération des mesures images dans la compensation par faisceaux. Pour ce faire, nous avons testé différentes valeurs d'incertitude des mesures (chaque observation se voyant attribuer un poids de $1 /$ Incertitude $e_{\text {tra }}^{2}$ et différents seuils pour limiter le poids d'une catégorie de mesures (points homologues - mesures terrain des points d'appui - mesures images des points d'appui) via le paramètre $N b \max$ (voir équation 1 et figure 7). Pour les mesures terrain des points d'appui, ce sont les écarts-types sur la mesure des coordonnées qui sont utilisés pour chacun des points d'appui comme valeur d'incertitude (écart-type délivré dans le rapport de traitement du DGPS). Un facteur de multiplication leur est ensuite appliqué afin de modifier cette incertitude. L'analyse des résidus pour chacune des configurations de compensation par faisceaux nous a ensuite amenés à dégager des règles simples pour la bonne utilisation des mécanismes de pondération implémentés dans Apero.

$$
\text { Poid }_{\text {set }}=\frac{x * N b \max }{x+N b \max }
$$

où

Poid $_{\text {set }}$ est le poids attribué au jeu de mesures (points homologues, mesures terrain des points d'appui et mesures images des points d'appui);

$x \quad$ est le nombre d'observations du jeu de mesures;

NbMax est le seuil de pondération attribué à ce jeu de mesures.

La corrélation dense est ensuite effectuée en géométrie terrain (espace objet) afin d'obtenir un MNT d'une résolution de $1: 2$ de la résolution des images brutes. Les MNTs ont donc une résolution de $12.5 \mathrm{~cm}$ par pixel.

\section{Résultats}

\subsection{Calcul de l'orientation externe}

\subsubsection{Orientation relative}

Lors des acquisitions, certains objets mobiles tels que des ombres d'arbres, des oiseaux et des voitures ont en-

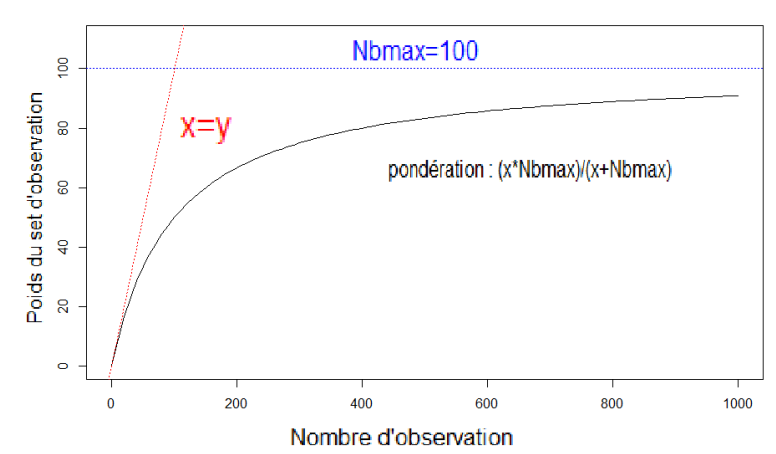

FIguRE 7: Représentation visuelle de l'un des trois mécanismes de pondération d'observations hétérogènes implémenté dans Apero: pondération d'un jeu d'observations en limitant le poids du set à Nbmax (equation 1. L'objectif de ce mécanisme est de limiter l'impact d'un gros jeu d'observations (tel que 50 000 points homologues par exemple) sur la détermination de l'orientation d'un bloc d'images afin de donner plus d'importance à un autre jeu d'observations (25 points d'appui par exemple).

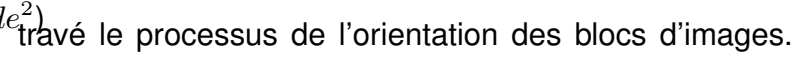
De plus, certaines images des acquisitions $t 2$ et $t 3$ de zones présentant très peu d'objets caractéristiques (uniquement le sol labouré) ne partagent pas suffisamment de points homologues avec les autres images et n'ont donc pas pu être orientés. On peut localiser sur les cartes présentées figure 35 les endroits où des images manquent dans les lignes de vol. Ce sont celles pour lesquelles l'orientation a échoué et qui ne sont dès lors pas utilisées dans la suite des traitements. Ces situations sont cependant très localisées et marginales, le résultat à l'échelle de la totalité du bassin versant étant très satisfaisant, avec des résidus moyens (erreur de reprojection des points homologues) entre 0.4 et 0.8 pixels $(t 1: 0.429, t 2: 0.768$ et $t 3: 0.426)$. L'acquisition $t 2$ présente de moins bons résultats en terme de résidus. De manière localisée pour les acquisitions $t 2$ et $t 3$, on note également la présence d'un tracteur labourant le sol, ce qui engendre des modifications de la scène entre les prises de vue successives.

\subsubsection{Orientation absolue avec utilisation des points d'ap- pui dans la compensation par faisceaux}

Conformément à notre hypothèse de base, les paramètres par défaut du logiciel Apero donnent trop d'importance aux mesures terrain des points d'appui, au détriment des mesures images. Cela s'explique, selon nous, par le fait que l'incertitude des mesures terrain, exprimée en mètre et de l'ordre de $0.015 \mathrm{~m}$, confère une pondération excessive à ces observations. Chaque observation se voit en effet attribuer un poids de $1 /$ Incertitude ${ }^{2}$. Par ailleurs, l' incertitude des mesures images des points d'appui, exprimée en pixels et représentative de la qualité du pointé du point d'appui sur l'image, est de l'ordre de 1 pixel au mieux pour nos jeux de données. C'est en augmentant l'incertitude des mesures terrain d'un facteur dix, entraînant donc une diminution de leur facteur de pondération, et en diminuant l'incertitude des mesures 
images de ces points d'appui à 0.5 pixel que les résultats se sont montrés les plus satisfaisants. En effet, les résidus sur les mesures terrain concordent avec la précision des relevés au DGPS, les résidus des mesures images étant dans la gamme de précision du marquage des cibles sur les images, et l'erreur-type (racine carrée de la moyenne des carrés des résidus) sur les points de vérification en validation croisée est la plus faible. Ces erreurs-types sont de $11,1 \mathrm{~cm}$ pour $t 1,18,3 \mathrm{~cm}$ pour $t 2$ et de $12,8 \mathrm{~cm}$ pour $t 3$. Une carte de ces résidus est présentée à la figure 8 pour chacune des acquisitions. La diminution ou l'augmentation du poids du jeu de mesures des points homologues via la valeur seuil Nbmax n'a quant à elle pas montré d'amélioration notable sur les résultats. La valeur par défaut de 100, qui a pour effet de limiter le poids des milliers de points homologues en-dessous de 100 , convient donc bien à nos jeux de données contenant une dizaine de points d'appui. C'est donc seulement l'équilibre entre pondération des mesures terrain et des mesures images qu'il a fallu contrôler afin d'obtenir une pondération optimale.

\subsection{Corrélation dense}

L'inspection visuelle des résultats de la corrélation dense montre qu'aucune aberration n'est à constater dans la reconstruction du relief, si ce n'est au-dessus des zones pour lesquelles certaines images n'ont pas été orientées (acquisition $t 2$ et $t 3$ ) et dans les ombres de la haie d'arbres traversant le bas du bassin versant dans sa longueur. Un apercu des MNSs (Modèle Numérique de Surface) est présenté à la figure 8 . Des détails tels que les lignes de labour sont nettement visibles sur les MNT, et les axes de ruissellement sont évidents à localiser.

\subsection{Détection de changement de relief par compa- raison des modèles numériques de terrain}

Les résultats de l'acquisition $t 2$ étant les moins satisfaisants en terme de résidus sur les points homologues, de résidus sur les points d'appui, ainsi qu'en terme de disposition des points d'appui, seuls les MNTs des acquisitions $t 1$ et $t 3$ ont fait l'objet d'une comparaison. La soustraction du MNT $t 1$ au MNT $t 3$ montre que les différences moyennes et maximales entre les deux modèles sont dans des gammes de valeurs plausibles. Après l'exclusion des zones présentant de la végétation à l'une des deux dates $t 1$ et $t 3$ et des zones s'écartant trop des points d'appui (extérieur du bassin versant), la carte des différences correspond au changement de relief s'étant produit durant les deux années séparant les deux acquisitions. Mises à part les lignes de labour qui sont bien présentes sur cette carte de changement de relief, c'est surtout l'érosion en ravine le long de certains axes de ruissellement qui ressort de l'analyse visuelle. La figure 9 montre un agrandi sur cette carte de changement de relief, et met en évidence ce changement de relief d'une magnitude de $30 \mathrm{~cm}$ en altimétrie.
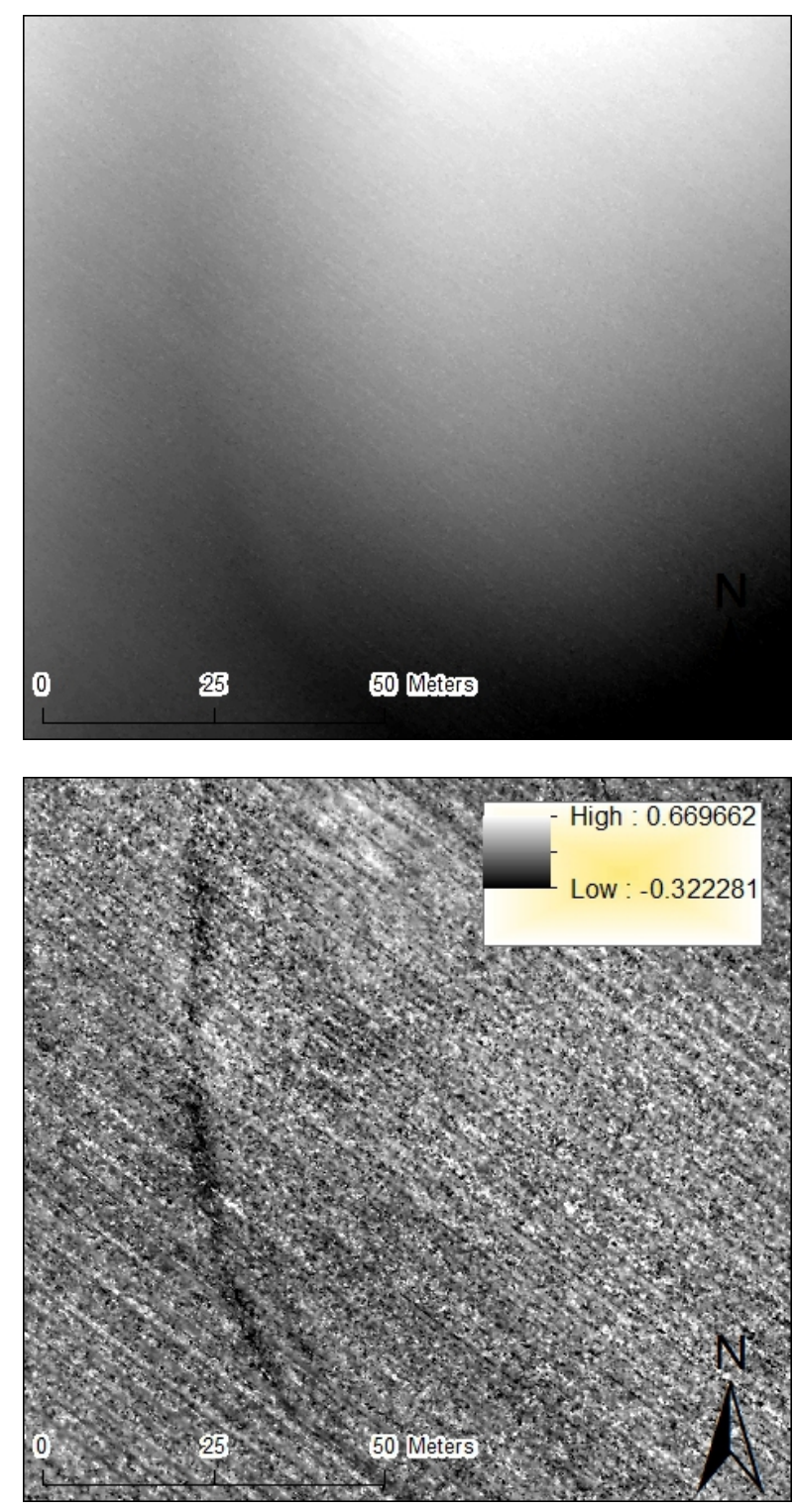

FIGURE 9: Une érosion en ravine détectée par soustraction du MNT 2013 au MNT 2011. En haut : le relief de la zone en 2013. En bas : la carte des différences, illustrant la ravine. Les lignes axées nord-ouest/sud-est sont dues au labour du sol. 

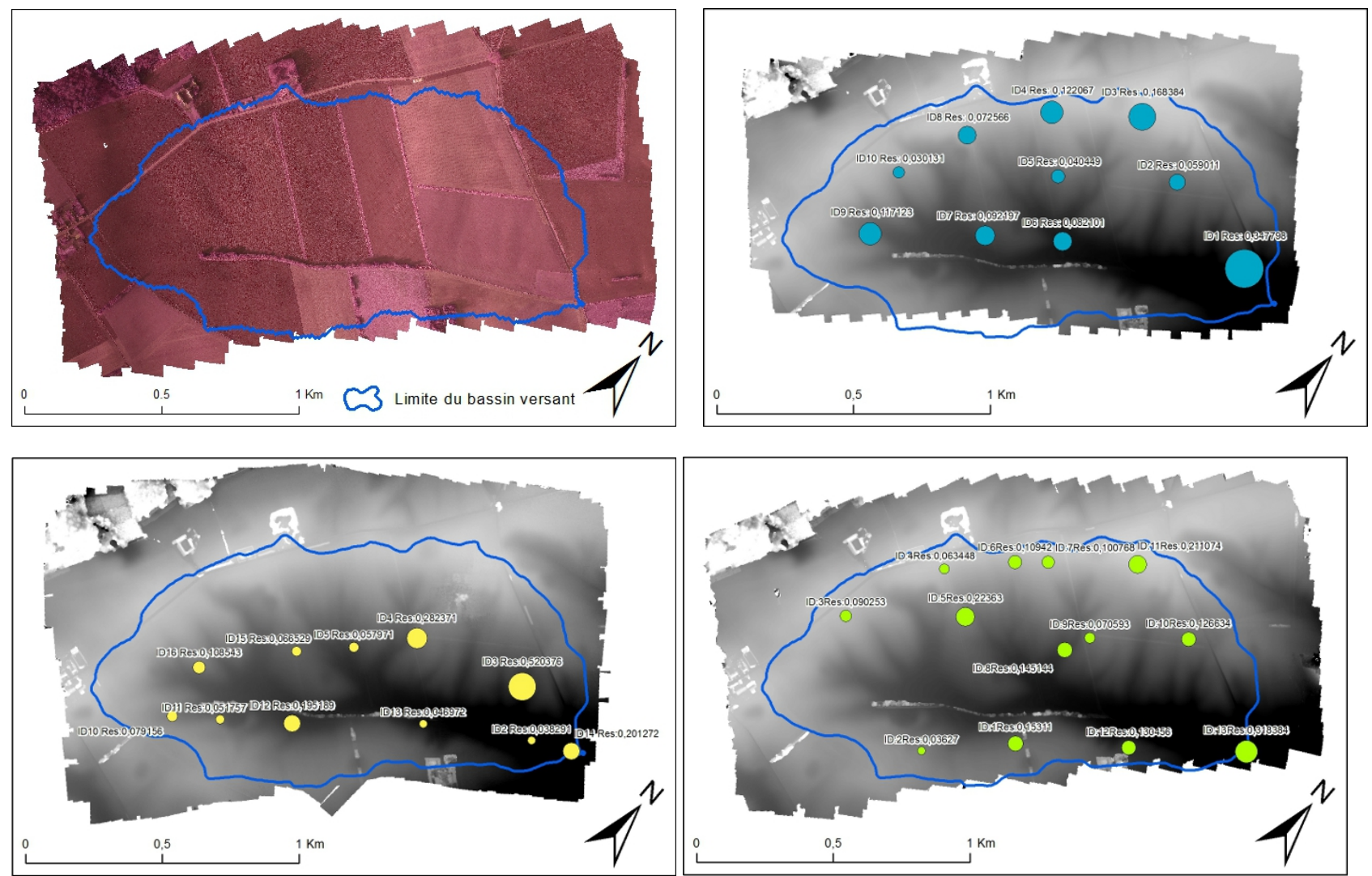

FIGURE 8: La série temporelle de Modèles Numériques de Terrain photogrammétriques du bassin versant agricole de Haute-Heuval. La résolution est de $12 \mathrm{~cm} /$ pixel. En haut à gauche : l'orthophotographie fausse couleur du bassin versant. En haut à droite : le MNT et les résidus sur les points de vérification de l'année 2011. En bas à gauche : le MNT et les résidus sur les points de vérification de l'année 2012. En bas à droite : le MNT et les résidus sur les points de vérification de l'année 2013. 


\section{Conclusion}

Cette recherche a abouti à une méthodologie d'acquisition et de traitements utilisée pour détecter des érosions et dépôts de manière très fine (voir figure 9 ). En particulier, l'orientation du bloc d'images par compensation par faisceaux avec points d'appui, effectuée avec les outils de la suite MICMAC, à savoir Apero, a fait l'objet de divers tests qui ont mis en évidence l'impact primordial de la pondération des observations hétérogènes sur la qualité des résultats. Dans Apero, il est primordial de trouver un équilibre entre le poids donné aux mesures terrain des points d'appui et aux mesures images de ces mêmes points d'appui.

Les points de contrôle au sol ont été utilisés en validation croisée comme points de vérification afin de quantifier la précision de géoréférencement. Bien que l'appareil photographique ait été précalibré, l'orientation interne est réajustée (autocalibration) en utilisant ces mêmes points d'appui. La comparaison de la série temporelle de MNTs à très haute résolution $(12.5 \mathrm{~cm} /$ pixel) a montré la possibilité de localiser des changements de relief de l'ordre de $30 \mathrm{~cm}$ (érosion). Ces changements subtils de relief seront utilisés comme base d'une réflexion portant sur l'impact des différentes cultures, de la présence de bandes herbeuses et de haies sur l'érosion du sol en milieu agricole.

\section{Remerciements}

Nos remerciements vont aux pilotes de l'unité de Gestion des Ressources forestières et des Milieux naturels : Alain Monseur et Cédric Geerts. Les relevés au DGPS ont été effectué par Mr Gilles Swerts, à qui nous adressons également nos remerciements. Nous exprimons notre reconnaissance à la Direction Générale des Transports Aériens, représentée en la personne de Erika Billen, ainsi que la commune de Court-Saint-Etienne pour avoir délivré les autorisations nécessaires aux opérations avec un drone. Enfin, nous remercions Mr Olivier Lisein pour sa contribution à l'amélioration de cet article.

\section{Bibliographie}

\section{Références}

Barneveld, R. J., Seeger, M., Maalen-Johansen, I., 2013. Assessment of terrestrial laser scanning technology for obtaining high-resolution DEMs of soils. Earth Surface Processes and Landforms 38 (1), 90-94.

URL http://onlinelibrary.wiley.com/doi/10. $1002 /$ esp.3344/full

Brubaker, K. M., Myers, W. L., Drohan, P. J., Miller, D. A., Boyer, E. W., 2013. The use of LiDAR terrain data in characterizing surface roughness and microtopography. Applied and Environmental Soil Science 2013.

URL http://www.hindawi.com/journals/aess/ 2013/891534/abs/

Colomina, I., Molina, P., juin 2014. Unmanned aerial systems for photogrammetry and remote sensing : A review. ISPRS Journal of Photogrammetry and Remote Sensing 92, 79-97. URL http://www.sciencedirect.com/science/ article/pii/s0924271614000501
d'Oleire Oltmanns, S., Marzolff, I., Peter, K. D., Ries, J. B., 2012. Unmanned aerial vehicle (UAV) for monitoring soil erosion in morocco. Remote Sensing 4 (11), 3390-3416.

URL http://www.mdpi.com/2072-4292/4/11/3390/ htm

Fonstad, M. A., Dietrich, J. T., Courville, B. C., Jensen, J. L., Carbonneau, P. E., 2013. Topographic structure from motion: a new development in photogrammetric measurement. Earth Surface Processes and Landforms 38 (4), 421-430.

URL http://onlinelibrary.wiley.com/doi/10. 1002/esp.3366/abstract

Hugenholtz, C. H., Moorman, B. J., Riddell, K., Whitehead, K., juin 2012. Small unmanned aircraft systems for remote sensing and earth science research. Eos, Transactions American Geophysical Union 93 (25), 236-236.

URL http://onlinelibrary.wiley.com/doi/10. 1029/2012E0250005/abstract

Javernick, L., Brasington, J., Caruso, B., mai 2014. Modeling the topography of shallow braided rivers using structure-frommotion photogrammetry. Geomorphology 213, 166-182.

URL http://www.sciencedirect.com/science/ article/pii/S0169555X14000245

Lejot, J., Delacourt, C., Piégay, H., Fournier, T., Trémélo, M.-L., Allemand, P., octobre 2007. Very high spatial resolution imagery for channel bathymetry and topography from an unmanned mapping controlled platform. Earth Surface Processes and Landforms 32 (11), 1705-1725.

URL http://65.54.113.26/Publication/39388299

Lisein, J., Pierrot-Deseilligny, M., Bonnet, S., Lejeune, P., novembre 2013. A photogrammetric workflow for the creation of a forest canopy height model from small unmanned aerial system imagery. Forests 4 (4), 922-944. URL http://www.mdpi.com/1999-4907/4/4/922

Lucieer, A., Jong, S., Turner, D., 2014. Mapping landslide displacements using structure from motion (SfM) and image correlation of multi-temporal UAV photography. Progress in Physical Geography 38 (1), 97-116.

Mancini, F., Dubbini, M., Gattelli, M., Stecchi, F., Fabbri, S., Gabbianelli, G., 2013. Using unmanned aerial vehicles (UAV) for high-resolution reconstruction of topography : The structure from motion approach on coastal environments. Remote Sensing 5 (12), 6880-6898. URL http://www.mdpi.com/2072-4292/5/12/6880/ htm

Niethammer, U., James, M., Rothmund, S., Travelletti, J., Joswig, M., 2012. UAV-based remote sensing of the super-sauze landslide : Evaluation and results. Engineering Geology 128, 2-11.

URL http://www.sciencedirect.com/science/ article/pii/s0013795211000755

Ouédraogo, M. M., Degré, A., Debouche, C., Lisein, J., juin 2014. The evaluation of unmanned aerial system-based photogrammetry and terrestrial laser scanning to generate DEMs of agricultural watersheds. Geomorphology 214, 339-355.

URL http://www.sciencedirect.com/science/ article/pii/S0169555X14000993

Peter, K. D., d'Oleire Oltmanns, S., Ries, J. B., Marzolff, I., Ait Hssaine, A., février 2014. Soil erosion in gully catchments affected by land-levelling measures in the souss basin, morocco, analysed by rainfall simulation and UAV remote sensing data. CATENA 113, 24-40.

URL http://www.sciencedirect.com/science/ article/pii/S0341816213002361

Pierrot-Deseilligny, M., 2014. Micmac documentation : MicMac, apero, pastis and other beverages in a nutshell! URL http://logiciels.ign.fr/?-Micmac, 3-

Pierrot-Deseilligny, M., Clery, I., 2011. Apero, an open source 
bundle adjustment software for automatic calibration and orientation of set of images. Dans : Proceedings of the ISPRS Symposium, 3DARCH11. pp. 1-8.

Pierrot-Deseilligny, M., Paparoditis, N., 2006. A multiresolution and optimization-based image matching approach : An application to surface reconstruction from SPOT5-HRS stereo imagery. Int. Archives of Photogrammetry, Remote Sensing and Spatial Information Sciences 36 (1/W41).

Remondino, F., Pizzo, S. D., Kersten, T. P., Troisi, S., janvier 2012. Low-cost and open-source solutions for automated image orientation - a critical overview. Dans : Ioannides, M., Fritsch, D., Leissner, J., Davies, R., Remondino, F., Caffo, R. (Eds.), Progress in Cultural Heritage Preservation. No. 7616 dans Lecture Notes in Computer Science. Springer Berlin Heidelberg, pp. 40-54.

URL http://link.springer.com/chapter/10. 1007/978-3-642-34234-9_5

Saavedra, C., 2005. Estimating spatial patterns of soil erosion and deposition in the Andean region using geo-information techniques: A case study in Cochabamba, Bolivia. Wageningen Universiteit.

URL http://library.wur.nl/WebQuery/clc/ 1783953

Slattery, M. C., Gares, P. A., Phillips, J. D., 2002. Slope-channel linkage and sediment delivery on north carolina coastal plain cropland. Earth Surface Processes and Landforms 27 (13), 1377-1387.

URL http://onlinelibrary.wiley.com/doi/10. 1002/esp.436/abstract

Snavely, N., Seitz, S. M., Szeliski, R., novembre 2008. Modeling the world from internet photo collections. International Journal of Computer Vision 80 (2), 189-210.

URL

http://dx.doi.org/10.1007/

Stroosnijder, L., 2005. Measurement of erosion : is it possible? Catena 64 (2), 162-173.

URL http://www.sciencedirect.com/science/ article/pii/S0341816205001268

Stumpf, A., Malet, J.-P., Kerle, N., Niethammer, U., Rothmund, S., mars 2013. Image-based mapping of surface fissures for the investigation of landslide dynamics. Geomorphology $186,12-27$.

URL http://www.sciencedirect.com/science/ article/pii/s0169555X12005594

Tarolli, P., juillet 2014. High-resolution topography for understanding earth surface processes : Opportunities and challenges. Geomorphology 216, 295-312.

URL http://www.sciencedirect.com/science/ article/pii/s0169555X14001202

Tournadre, V., Pierrot-Deseilligny, M., Faurec, P. H., 2014. UAV PHOTOGRAMMETRY TO MONITOR DYKES-CALIBRATION AND COMPARISON TO TERRESTRIAL LIDAR. ISPRS-International Archives of the Photogrammetry, Remote Sensing and Spatial Information Sciences 1 (1), 143-148.

Warner, W. S., 1995. Mapping a three-dimensional soil surface with hand-held $35 \mathrm{~mm}$ photography. Soil and Tillage Research 34 (3), 187-197.

URL http://www.sciencedirect.com/science/ article/pii/0167198795004622

Westoby, M. J., Brasington, J., Glasser, N. F., Hambrey, M. J., Reynolds, J. M., 2012. 'Structure-fromMotion'photogrammetry : a low-cost, effective tool for geoscience applications. Geomorphology 179, 300-314.

URL http://www.sciencedirect.com/science/ article/pii/S0169555X12004217

Wu, C., 2014. Critical configurations for radial distortion selfcalibration. CVPR. 